\title{
Das Hybride Wahlmodell und seine Anwendung im Marketing
}

\author{
Till Dannewald* \\ Henning Kreis* \\ Nadja Silberhorn*
}

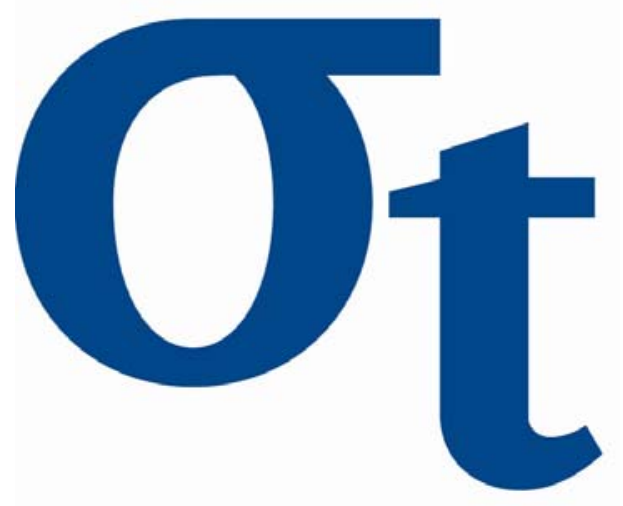

* Humboldt-Universität zu Berlin Germany

This research was supported by the Deutsche Forschungsgemeinschaft through the SFB 649 "Economic Risk". 


\title{
Das Hybride Wahlmodell und seine Anwendung im Marketing ${ }^{1}$
}

\author{
Till Dannewald, Henning Kreis, Nadja Silberhorn \\ Institut für Marketing, Humboldt-Universität zu Berlin \\ Spandauer Straße 1, 10099 Berlin \\ \{dannewald,kreis,silberhorn\}@wiwi.hu-berlin.de
}

\section{Zusammenfassung}

In klassischen Wahlmodellen wird davon ausgegangen, dass sich ein beobachtetes Verhalten durch einen nicht näher spezifizierbaren Evaluationsprozess des beobachteten Individuums ergibt. Ist die Aufdeckung dieses Prozesses von Interesse, stoßen reine Wahlmodelle schnell an ihre methodischen Grenzen, da psychologische Größen wie beispielsweise die Wahrnehmung von Konsumenten oder die Einstellung zu Produkten als nicht direkt messbare Variablen nicht ohne Weiteres in die Modellstruktur zu integrieren sind. Der kausalanalytische Ansatz bietet die Möglichkeit der Spezifikation nicht direkt messbarer Größen als latente Variablen und kann folglich Wahlmodelle sinnvoll ergänzen. Bislang werden von vielen Anwendern methodische Ansätze, die latente Variablen untersuchen, und klassische Wahlmodelle als unabhängig voneinander wahrgenommen und eingesetzt. In der vorliegenden Arbeit werden die Möglichkeiten der Integration von latenten Variablen in klassische Wahlmodelle herausgestellt sowie ein inhaltlicher Einstieg in die Modellierung von Hybriden Wahlmodellen geliefert. Zudem werden mögliche Anwendungsbereiche in der Marketingforschung skizziert.

\section{Summary}

Traditional choice models assume that observable behavior results from an unspecified evaluation process of the observed individual. When it comes to the revelation of this process mere choice models rapidly meet their boundaries, as psychological factors (e.g., consumers' perception or attitudes towards products) are not directly measurable variables and therefore cannot offhand be integrated within the model structure. The causal-analytic approach offers the possibility to specify not directly measurable factors as latent variables, and can thus reasonably supplement choice models. So far, methodological approaches investigating latent variables, and traditional choice models are perceived and applied independently of one another. In this paper the possibilities of an integration of latent variables into traditional choice models is pointed out, and an introduction into the modeling of hybrid choice models is provided. Furthermore, potential areas of application in marketing research are outlined.

\section{Schlüsselbegriffe}

Hybrides Wahlmodell, latente Variablen, Kausalmodell, Wahlmodell

\section{Keywords}

Hybrid choice model, latent variables, causal model, choice model

\section{JEL-Codes}

M30, C51, C10

\footnotetext{
${ }^{1}$ Finanziell gefördert durch die Deutsche Forschungsgemeinschaft (DFG) im SFB 649 „Ökonomisches Risiko“ und im Forschungsprojekt \#1952/1.
} 


\section{Einleitung}

Zahlreiche wissenschaftliche Forschungsrichtungen, insbesondere in den Bereichen Ingenieurwissenschaften, Psychologie und Ökonomie, sind mit der Analyse und Erklärung von Entscheidungs- und Auswahlprozessen befasst. Dabei werden sogenannte Wahlmodelle in den verschiedensten Anwendungsbereichen genutzt, um Erkenntnisse über den Ablauf von Entscheidungen zu gewinnen (vgl. McFadden 2000). Im Marketing finden diese Modelle insbesondere bei der Analyse von Kaufentscheidungsprozessen Beachtung (z. B. Chintagunta 1993; Guadagni/Little 1983; Kamakura/Russell 1989). Wird der Produktkauf als stochastischer Entscheidungsprozess verstanden (v.a. im Bereich der Psychologie), lassen sich Auswahlwahrscheinlichkeiten für jede Alternative mit Modellen des Bradley-Terry-Luce (BTL) Typs bestimmen. Bei Wahlmodellen, die auf der Zufallsnutzentheorie basieren, steht die Annahme, dass Konsumenten immer individualnutzenmaximierend agieren, im Vordergrund. Es wird davon ausgegangen, dass sich ein beobachtetes Verhalten durch einen nicht näher spezifizierbaren Evaluationsprozess des beobachteten Individuums ergibt. In den Standardmodellen optimiert der Konsument seine Wahlentscheidung innerhalb einer für den Forscher nicht einsehbaren „Black Box“ (vgl. Morikawa et al. 2002).

Der Nobelpreisträger Daniel McFadden stellt fest, dass „[the] empirical study of economic behavior would benefit from closer attention to how perceptions are formed and how they influence decision-making“ (McFadden 1997). Ist die Aufdeckung dieses Prozesses von Interesse, stoßen reine Wahlmodelle schnell an ihre methodischen Grenzen, da psychologische Größen wie beispielsweise die Wahrnehmung von Konsumenten oder die Einstellung zu Produkten als nicht direkt messbare Variablen nicht ohne Weiteres in die Modellstruktur zu integrieren sind. Gerade diese Einflussgrößen spielen jedoch in Kaufentscheidungsprozessen häufig die entscheidende Rolle (Ben-Akiva et al. 2002a, 1999). Entscheidungsmodelle des Konsumentenverhaltens (z. B. nach Blackwell et al. 2006) stellen den Ablauf des Entscheidungsprozesses beim Kauf in den Mittelpunkt und versuchen, diesen Prozess durch Zerlegung in einzelne Stufen zu strukturieren. Der kausalanalytische Ansatz bietet die Möglichkeit der Spezifikation nicht direkt messbarer Größen als latente Variablen und kann folglich die Wahlmodelle in der Analyse von Kaufentscheidungsprozessen sinnvoll ergänzen.

Die Aufnahme psychologischer Faktoren lässt eine realistischere Betrachtung des Entscheidungs- und Auswahlprozesses zu, wodurch ein besseres Verständnis des Konsumentenverhaltens ermöglicht wird. Die Integration unbeobachtbarer Einflussgrößen des Konsu- 
mentenverhaltens in Wahlmodelle mittels latenter Variablen wurde bislang weitgehend vernachlässigt (Ashok et al. 2002). Dabei bietet die Berücksichtigung latenter Variablen eine wesentliche Möglichkeit, den Einsatz von Marketing-Mix-Instrumenten gezielter auf Kundenbedürfnisse zuzuschneiden.

Bislang werden von vielen Anwendern methodische Ansätze, die latente Variablen untersuchen (z.B. mittels LISREL- oder PLS-Ansatz), und klassische Wahlmodelle als unabhängig voneinander wahrgenommen und eingesetzt. Der originäre Fokus dieser Arbeit besteht in diesem Zusammenhang darin, die Möglichkeit der Verknüpfung dieser Forschungsrichtungen einem breiten Publikum aufzuzeigen. Vor allem sollen dabei die Vorzüge der Integration von latenten Variablen in ein klassisches Wahlmodell herausgestellt sowie ein inhaltlicher Einstieg in die Modellierung eines Hybriden Wahlmodells geliefert werden. Dadurch wird dem Marktforscher die Möglichkeit eröffnet, einen tieferen Einblick in die Entscheidungsprozesse des Konsumenten zu erlangen und somit die o. a. „Black Box“ zu öffnen. Zudem werden mögliche Anwendungsbereiche in der Marketingforschung skizziert.

Der Beitrag gliedert sich vor diesem Hintergrund wie folgt: Kapitel 2 erörtert zentrale Bereiche der Entscheidungsforschung vor dem Hintergrund des Einsatzes von Hybriden Wahlmodellen. Das nachfolgende Kapitel widmet sich der Methodik und beschreibt unterschiedliche Möglichkeiten, unbeobachtbare Einflussgrößen in ein Wahlmodell zu integrieren. Kapitel 4 diskutiert Erweiterungsmöglichkeiten des vorgestellten Modellrahmens, während Kapitel 5 mögliche Anwendungsbereiche für den Marktforscher zeigt. Der Beitrag schließt mit einer Diskussion in Kapitel 6.

\section{Einordnung in zentrale Bereiche der Entscheidungsforschung}

Abb. 1 verdeutlicht die verschiedenen Bereiche der Entscheidungsforschung (vgl. Ben-Akiva et al. 2002a), auf die im Folgenden jeweils kurz eingegangen wird. 


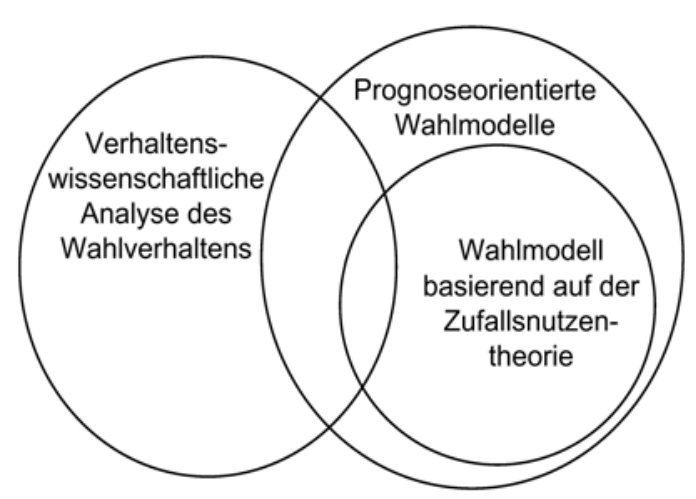

Abb. 1: Bereiche der Entscheidungsforschung (vgl. Ben-Akiva et al. 2002a)

Prognoseorientierte Wahlmodelle gehen von einem Entscheidungsverhalten aus, das weitgehend durch Regelmäßigkeiten, im Sinne eines kausalen Zusammenhangs zwischen dem Vorliegen bestimmter Eigenschaften und dem daraus resultierenden Verhalten, geprägt ist. Diese werden in quantitativen Modellen genutzt, um mit einer bestimmten Wahrscheinlichkeit Voraussagen für die Zukunft zu machen und Entscheidungen zu treffen. Hierbei werden üblicherweise „revealed preferences“ (RP), also das tatsächliche Marktverhalten bzw. die tatsächliche Auswahlentscheidung, sowie beobachtbare Attribute der zur Auswahl stehenden Alternativen und sozio-ökonomische Charakteristika des Entscheidungsträgers zur Modellschätzung herangezogen (Morikawa et al. 2002). Der Entscheidungsprozess wird dabei als „black box“ betrachtet, in den die Eigenschaften der Alternativen sowie individuelle Charakteristika des Entscheidungsträgers als Input einfließen, und aus dem die beobachtete Wahlentscheidung als Output hervorgeht (Ben-Akiva et al. 2002b).

Innerhalb dieses Bereichs sind auf der Zufallsnutzentheorie basierende Wahlmodelle ein leistungsstarkes Instrument, um die systematischen Aspekte des Verhaltens abzubilden. Zentrale Annahme dieser sog. random utility maximization (RUM) Modelle ist, dass der Entscheidungsträger mit einer begrenzten Anzahl sich untereinander ausschließender Alternativen konfrontiert ist und er aus dieser Menge diejenige Alternative auswählt, die ihm den größten Nutzen stiftet (Gopinath et al. 2004). Der Nutzen einer jeden Alternative setzt sich dabei additiv aus einer deterministischen und einer stochastischen Komponente zusammen. Mit diesem Ansatz kann die Auswahlwahrscheinlichkeit jeder Alternative bestimmt werden.

Neben den prognoseorientierten Wahlmodellen, die auf der Zufallsnutzentheorie basieren, spielen vor allem Wahlmodelle mit stochastischen Entscheidungsregeln eine große Rolle. Das BTL-Modell geht von einem intrinsisch probabilistischen Kaufverhalten aus, so dass der Konsument nicht zwingend die Alternative mit dem größten Individualnutzen auswählt. 
NBD-Modelle gehen wiederum davon aus, dass das Konsumentenverhalten ein stochastischer Prozess ist.

Zur Aufdeckung von Merkmalen des Entscheidungs- und Auswahlverhaltens im Sinne einer verhaltenswissenschaftlichen Analyse des Wahlverhaltens wird vorrangig mit Kausalmodellen (Structural Equation Models (SEM)) gearbeitet, bei denen die kausale Wirkung von Konstrukten beim Entscheidungsprozess im Mittelpunkt steht (Für einen Überblick zur Kausalanalyse siehe Hildebrandt/Homburg 1998). Als abhängige Variable wird jedoch häufig nicht das direkte Verhalten, sondern lediglich die abgefragte Verhaltensintention der Individuen eingesetzt. Psychometrische Modelle befassen sich traditionell mit Messfehlern und latenten Konstrukten, die über multiple Indikatoren gemessen werden (Muthén 2002).

In $A b b$. 2 werden der traditionelle Ansatz zur Modellierung von Wahlverhalten und der um latente Einflussgrößen erweiterte Ansatz gegenübergestellt. Im erweiterten Ansatz wird der Entscheidungsprozess nicht länger als „black box“ betrachtet, sondern es wird versucht, unterschiedliche Quellen einer Variation im Entscheidungsprozess zu berücksichtigen und damit diese „black box“ zu öffnen. Die Abbildung von Kausalzusammenhängen ermöglicht die Zerlegung des Entscheidungsprozesses. Dieser wiederum kann individuenspezifisch hinsichtlich seiner Komplexität, seines immanenten Risikos, seines Kontexts, sowie hinsichtlich der sozialen Situation des Entscheidungsträgers, dessen Motivation oder Vorwissen variieren (Ben-Akiva et al. 1999). Latente Variablen können unbeobachtbare Heterogenität und individuen- oder segmentspezifische Variationsquellen repräsentieren (Muthén 2002). 


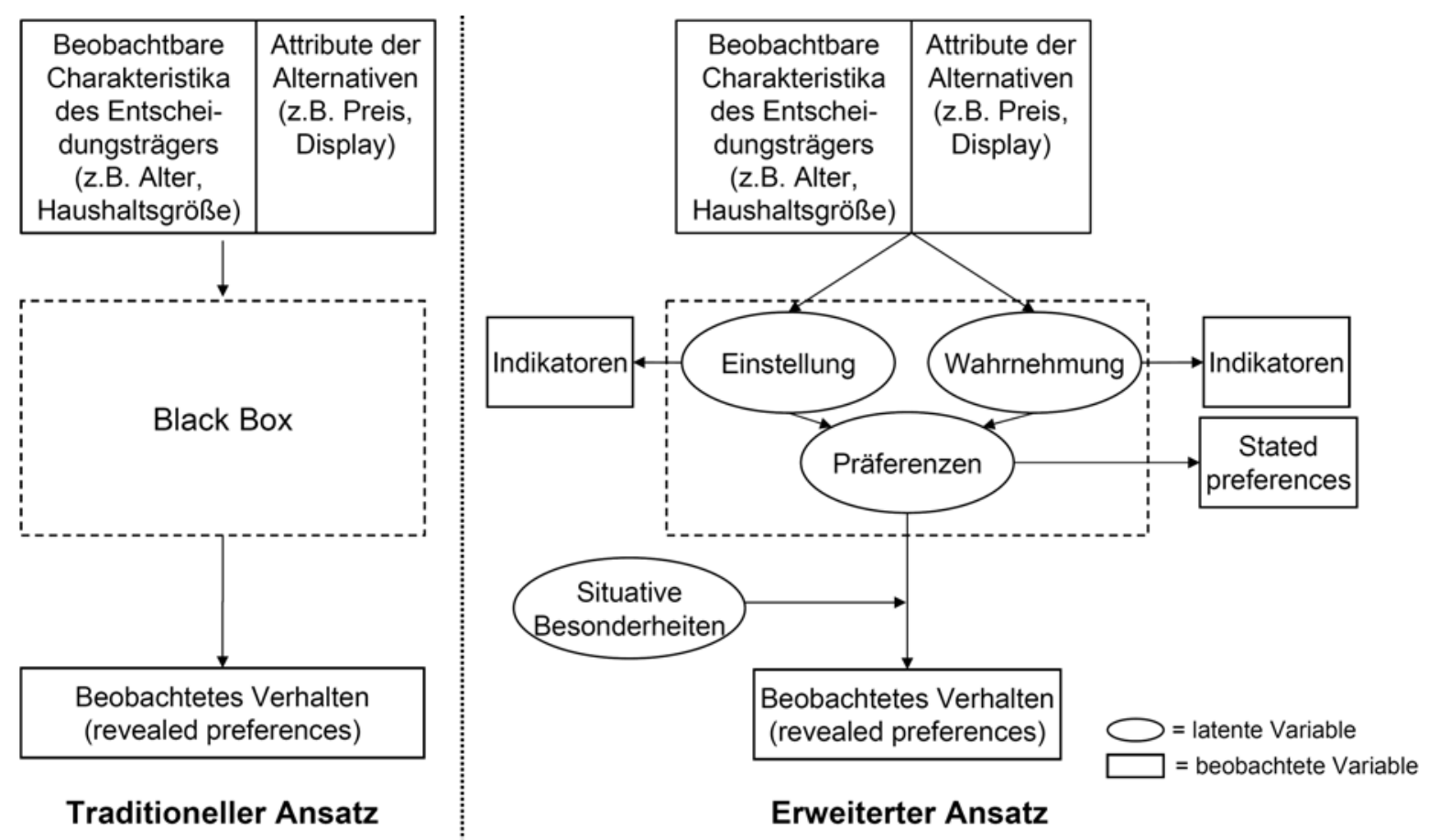

Abb. 2: Ansätze zur Modellierung von Wahlverhalten (in Anlehnung an Morikawa et al. 2002)

Ziel dieses Beitrags ist es, die verhaltenswissenschaftliche Analyse von Entscheidungsprozessen und die auf Prognose gerichtete Modellierung von Auswahlentscheidungen in einen umfassenden Modellansatz zu integrieren (Abb. 3). Ben-Akiva et al. (1999) betonen die Notwendigkeit weitergehender Untersuchungen im Hinblick auf die Integration von Wahlmodellen und Modellen mit latenten Konstrukten. Die Kluft zwischen Wahlmodellen und Kausalmodellen existiert, weil Forscher im Bereich der Modellierung von diskreten Wahlentscheidungen auf die Abbildung des Zusammenhangs zwischen exogenen Inputvariablen und der Wahlentscheidung des Entscheidungsträgers fokussiert sind, während Forscher im Bereich Verhaltenswissenschaften das Wesen des Entscheidungsprozesses und das Zustandekommen von Entscheidungen verstehen wollen (Walker 2001). In der experimentellen Forschung können lediglich die dem Entscheidungsverhalten zu Grunde liegenden exogenen Inputvariablen manipuliert werden. Im Marketing ist jedoch in vielen Fällen davon auszugehen, dass die Entscheidung für eine bestimmte Auswahlalternative auch durch nicht produktbezogene Attribute beeinflusst wird. Auf welche Art und Weise kann im Modell den „weichen“ Attributen, wie z. B. Einstellungen und Wahrnehmungen, Rechnung getragen werden (Ashok et al. 2002)? 


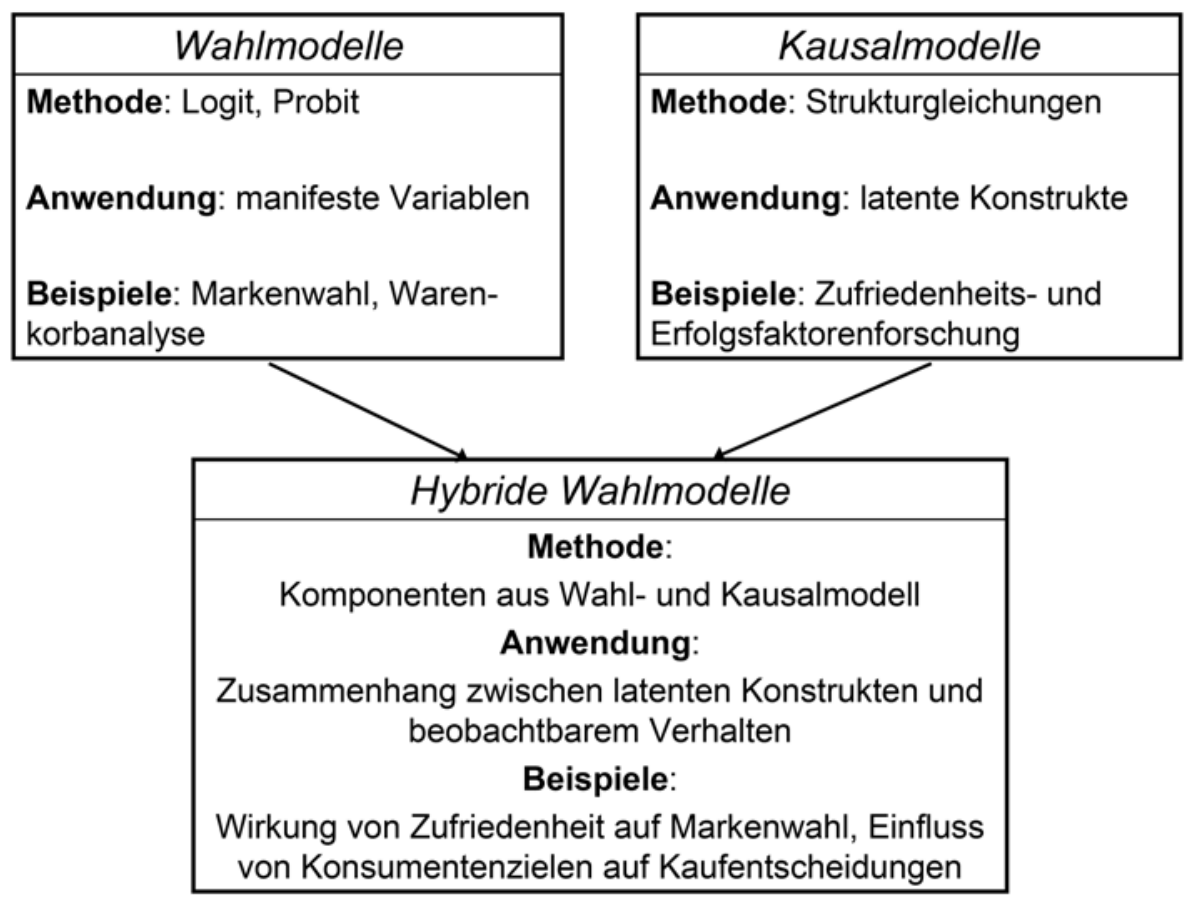

Abb. 3: Forschungsbereiche

Aktuelle Publikationen zur Modellierung von diskreten Wahlentscheidungen (Wahlmodelle) lassen sich anhand der verwendeten Methodik in zwei große Gruppen einteilen: LogitModelle (Guadagni/Little 1983; Hruschka et al. 1999; Niraj et al. 2007; Russell/Petersen 2000; Song/Chintagunta 2007) und Probit-Modelle (Ainslie/Rossi 1998; Seetharaman et al. 1999). Beide Ansätze beruhen auf der Theorie der Nutzenmaximierung und unterscheiden sich in der Spezifizierung der Fehlerterme. An dieser Stelle sei lediglich auf grundlegende (Hausman/Wise 1978; McFadden 1980, 1974) und in die Methodik einführende (BenAkiva/Lerman 1985; Chib/Greenberg 1998; Train 2003) Literatur verwiesen.

Der integrierte Modellansatz des Hybriden Wahlmodells kann in einer signifikanten Verbesserung des Goodness-of-Fit gegenüber Wahlmodellen ohne Berücksichtigung latenter Variablen resultieren. Dies ist jedoch stark abhängig vom Modell und der jeweiligen Anwendung (Ben-Akiva et al. 1999): In einigen Fällen ist ein substanzieller Unterschied im Vergleich zu einfachen Wahlmodellen zu finden, insbesondere, wenn der Einfluss der latenten Variable auf die Entscheidung erheblich ist. Jedoch sind auch Fälle denkbar, in denen der Einfluss gering ist und somit keine signifikante Verbesserung gegenüber klassischen Wahlmodellen erzielt werden kann. Um die Vergleichbarkeit der Modelle hinsichtlich ihrer Gütemaße bzw. Informationskriterien zu gewährleisten, muss bei der Analyse eines klassischen Wahlmodells parallel auch das (eigenständige) Kausalmodell mitgeschätzt werden. Die statistische Effizienz kann mittels geeigneter Testkriterien (Goodness-of-Fit-Maße, Chi-Quadrat-Differenzentest, 
etc.) oder entsprechender Informationskriterien (AIC, BIC, CAIC) überprüft werden. Jedoch kann mit diesen Kriterien lediglich die Anpassung des Modells an den untersuchten Datensatz beurteilt werden. Um Aussagen über die tatsächliche Prognosegüte treffen zu können, sollte außerdem die inhaltliche Konsistenz validiert werden. Zusätzlich ist auch eine Güteprüfung des Modells mit einem weiteren unabhängigen Datensatz bzw. über ein Holdout-Sample zu empfehlen. Darüber hinaus stellt das Hybride Wahlmodell eine realitätsnähere und verhaltenswissenschaftlich befriedigendere Abbildung des Entscheidungsverhaltens dar (Walker 2001).

McFadden (1986) gilt auf diesem Forschungsgebiet als Vorreiter und hat bereits vor zwanzig Jahren die Aufnahme von latenten Variablen in diskrete Wahlmodelle gefordert. Diesen Forderungen und Vorschlägen folgend, haben in erster Linie Moshe Ben-Akiva und seine Kollegen an der modelltechnischen Umsetzung gearbeitet (Ashok et al. 2002; Ben-Akiva et al. 1999, 2002a, 2002b; Bolduc et al. 2005; Gopinath et al. 2004; Walker 2001; Walker/BenAkiva 2002).

\section{Methodik}

Zur expliziten Erfassung psychologischer Faktoren in der Modellierung von diskreten Wahlentscheidungen werden unterschiedliche Ansätze verfolgt. Zunächst werden einige dieser Ansätze kurz charakterisiert sowie ihre Schwachstellen identifiziert, bevor im Anschluss der Vorschlag des Hybriden Wahlmodells von Walker \& Ben-Akiva (2002) ausführlich vorgestellt wird.

Ein erster Ansatz bezieht Indikatoren psychologischer Faktoren (z. B. Antworten auf Befragungen zu Einstellung und Wahrnehmung) direkt in die Nutzenfunktion ein (Abb. $4 a$, vgl. Koppelman/Hauser 1979). Die Indikatoren können jedoch nicht als auslösend (kausal) für die Entscheidung interpretiert werden, sondern repräsentieren lediglich z.B. die jeweilige Einstellung zum Untersuchungsgegenstand. Folglich liefern sie streng genommen keinen Nutzenbeitrag in der der Entscheidung zu Grunde liegenden Nutzenfunktion. Ein Wahlmodell, in dessen Nutzenfunktion die Indikatoren direkt als erklärende Größen einfließen, ist zur Prognose nicht geeignet, da in der Regel die notwendigen Daten nicht vorliegen.. Darüber hinaus ignoriert die direkte Aufnahme der Indikatoren (d. h. manifeste Items) als erklärende Variablen die Tatsache, dass die Indikatoren mit Messfehlern behaftet sind (Ben-Akiva et al. 2002b). Diese gehen in den Fehlerterm des Wahlmodells ein, ohne jedoch die jeweiligen Verteilungsannah- 
men der beiden Fehlertermkomponenten zu berücksichtigen. Eine weitere Einschränkung dieser Vorgehensweise besteht in der Gefahr von Multikollinearität durch die Einführung von miteinander korrelierenden Indikatoren.

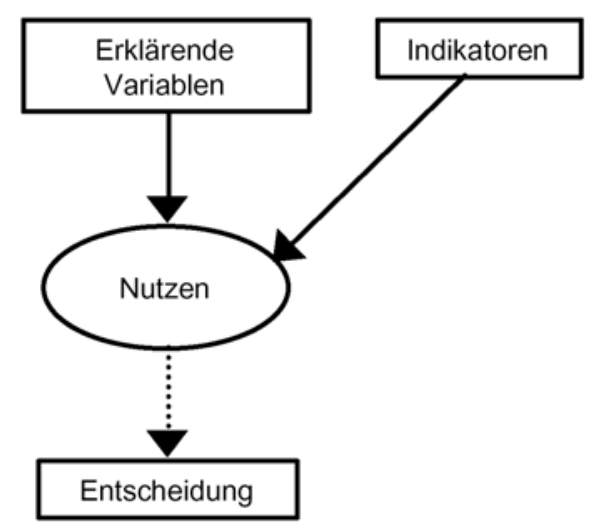

Abb. 4a: Wahlmodell mit direkt in den Nutzen einbezogenen Indikatoren (Ben-Akiva et al. 2002b)

Eine weitere, aus dem Bereich der Marktforschung entstammende Möglichkeit zur Berücksichtigung psychologischer Faktoren in Wahlmodellen besteht darin, latente Attribute direkt in die Nutzenfunktion einfließen zu lassen (Abb. 4b, vgl. Elrod/Keane 1995). Für diese latenten Attribute werden keine Indikatoren im klassischen Sinne zur Operationalisierung genutzt, sondern lediglich das beobachtete Wahlverhalten (vgl. Elrod/Keane 1995; Keane 1997). Morikawa et al. (2002) beispielsweise spezifizieren das latente Attribut „Komfort eines Transportmittels“ ausschließlich aus beobachtbaren Variablen wie etwa der Reiseklasse und der Dauer der Reise. Entsprechend sind hier latente Attribute alternativenspezifisch und über alle Individuen in einem Segment gleich, was das Modell restriktiv macht. Sie unterscheiden sich letztendlich nicht von anderen erklärenden Variablen, wie z.B. einer Promotionaktivität, die für alle Kunden identisch ist. Die fehlende Berücksichtigung von Wahrnehmungs- bzw. Einstellungsindikatoren bei der Schätzung ist ein weiterer Kritikpunkt dieser Modellklasse. Solche Indikatoren können nur nach der Analyse, also ex post, als Interpretationshilfe eingesetzt werden (Ben-Akiva et al. 2002b). 


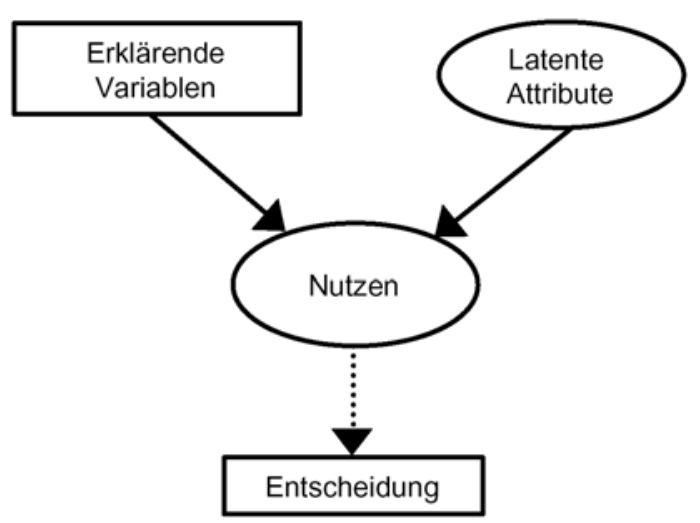

Abb. 4b: Modell mit latenten Attributen (Ben-Akiva et al. 2002b)

Um die Kritik der beiden geschilderten Verfahren ( $A b b .4 a$ und $4 b$ ) zu umgehen, wird häufig ein sequenzielles Verfahren ( $A b b .4 c$ ) eingesetzt, bei dem zunächst eine Faktoranalyse auf die Indikatoren durchgeführt wird (vgl. Madanat et al. 1995). In einem zweiten Schritt werden dann die angepassten latenten Variablen in die Nutzenfunktion eingefügt. Johansson et al. (2005) verwenden diesen Ansatz und zeigen, dass ein sequenzielles Verfahren das Wahlverhalten besser abbildet als das diskrete Wahlmodell. Allerdings lässt ein zweistufiger Ansatz die Kovariation zwischen manifesten Items und der Entscheidung unberücksichtigt (Ashok et al. 2002). Auch ist zu beachten, dass die angepassten latenten Variablen nicht messfehlerfrei sind. Aus diesem Grund muss die Auswahlwahrscheinlichkeit über die Verteilung der latenten Variablen integriert werden, um konsistente Schätzer zu erhalten (vgl. Morikawa 1989). Allerdings bleiben auch in diesem Fall die Schätzer ineffizient (Ashok et al. 2002; BenAkiva et al. 2002b) und können verzerrt sein (Walker et al. 2007).

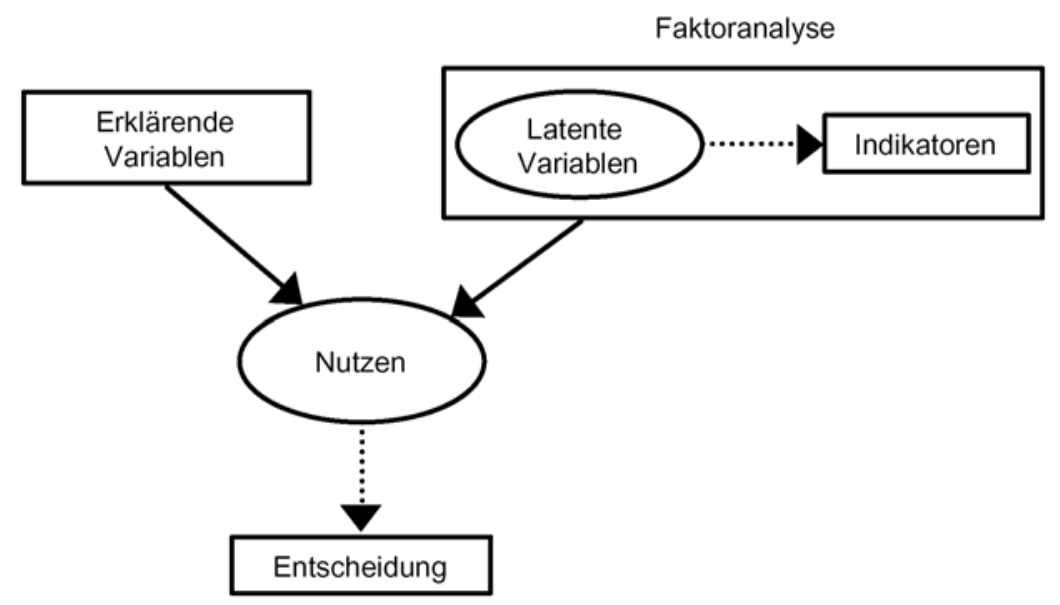

Abb. 4c: Sequenzielle Schätzung (Ben-Akiva et al. 2002b)

Um konsistente und effiziente Schätzer zu erhalten, müssen zwei Arten von Modellen (vgl. Abb. 3 und 5) in einen Modellansatz integriert werden: Wahlmodell und Kausalmodell. Die 
Methode erfordert die simultane Schätzung eines integrierten Mehrgleichungsmodells, das aus den Mess- und Strukturgleichungen des Wahl- und des Kausalmodells besteht.

Walker/Ben-Akiva (2002) stellen einen integrierten Modellansatz zur Einbettung latenter Variablen in Wahlmodelle vor ( $A b b$. 5). Während die latenten Konstrukte selbst unbeobachtbar sind, sind deren Auswirkungen auf Indikatoren beobachtbar. Die Indikatoren erlauben eine Identifikation der latenten Konstrukte und beinhalten Informationen und bieten Potenzial für größere Effizienz in der Modellschätzung, i.S.v. Parameterschätzungen mit geringen Standardfehlern (Ben-Akiva et al. 1999). Das Hybride Wahlmodell (Hybrid Choice Model (HCM)) geht demzufolge über das klassische zufallsnutzenmaximierende Wahlmodell hinaus und kann, wie in $A b b .5$ dargestellt, verhaltenswissenschaftliche Elemente über eine Kausalstruktur berücksichtigen (Ashok et al. 2002; Ben-Akiva et al. 2002a). Wahlmodelle können auf diese Weise mit der Einbeziehung kognitiver Vorgänge angereichert werden (Ben-Akiva et al. 2002b).

Den dem Entscheidungsprozess zu Grunde liegenden Verhaltenscharakteristika wird durch die Aufnahme latenter Variablen in das Modell Rechnung getragen. „Harte Informationen“ (z. B. sozio-ökonomische Charakteristika) werden mit „weichen Informationen“ bzgl. der Heterogenität der Grundgesamtheit (z. B. Indikatoren für schwer messbare psychologische Charakteristika wie Risikoaversion, Ungeduld usw.) verknüpft. Ziel ist es dabei, irrational erscheinendes Verhalten zu erklären und dabei durch Verknüpfung ökonomischer und psychologischer Daten einen Teil der unbeobachtbaren Heterogenität im Modell zu erfassen (BenAkiva et al. 2002a). 


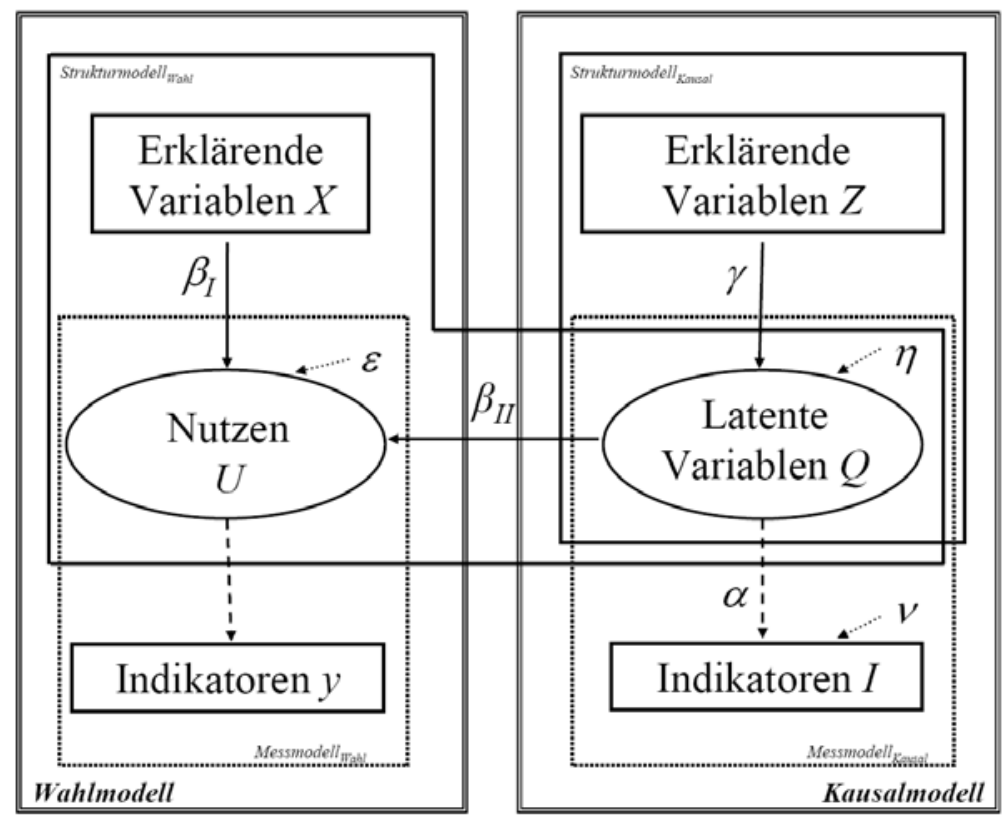

Abb. 5: Hybrides Wahlmodell als integrierter Modellansatz

Der integrierte Modellansatz kann verwendet werden, wenn wichtige kausale Variablen nicht direkt beobachtbar sind. Die zu grundlegende Idee besteht darin, latente Konstrukte explizit in das Modell einzubeziehen, um realistischere Verhaltensmodelle zu generieren (Walker 2001). Der latente Nutzen einer jeden Alternative wird als Funktion beobachtbarer erklärender Variablen $X$ mit zugehörigen Koeffizienten $\beta_{I}$, demografischer Eigenschaften $Z$ des Entscheidungsträgers und latenter Variablen $Q$ mit zugehörigen Koeffizienten $\beta_{I I}$ sowie einem Fehlerterm modelliert. Die demografischen Eigenschaften $Z$ können auch direkt in die Nutzenfunktion einfließen. Dies wurde aus Gründen der Übersichtlichkeit in $A b b .5$ vernachlässigt. Unter der Annahme der Nutzenmaximierungstheorie wählt der Konsument diejenige Alternative aus, die ihm den größten Nutzen stiftet. Die Wahl des Entscheidungsträgers wird angezeigt durch den Wahlindikator $y$, der die Werte 1 (Wahl) oder 0 (Nicht-Wahl) annimmt. Da die latenten Variablen unbeobachtbar sind, müssen sie mittels Indikatoren gemessen werden.

Das Wahlmodell und das Kausalmodell bestehen jeweils aus mindestens einer Strukturgleichung und mindestens einer Messgleichung. Die Strukturgleichung des Kausalmodells ist in diesem Zusammenhang durch Gleichung (1) gegeben.

$$
Q=h(Z ; \gamma)+\eta
$$


Das abhängige Konstrukt $Q$ wird durch die exogenen Einflussgrößen $Z$, die entsprechenden Pfadkoeffizienten $\gamma$, sowie den Störterm $\eta$ der endogenen latenten Variable bestimmt ${ }^{2}$. Das zur Umsetzung des Kausalmodells notwendige Messmodell kann mit den Faktorladungskoeffizienten $\alpha$ und den Messfehlern $v$ entsprechend Gleichung (2) beschrieben werden.

$$
I=g(Q ; \alpha)+v
$$

Die zweite Strukturgleichung eines Hybriden Wahlmodells wird durch die aus der Nutzentheorie bekannte Gleichung (3) beschrieben.

$$
U=V(X, Q ; \beta)+\varepsilon
$$

Im Unterschied zu einem klassischen Wahlmodell fließt hier zusätzlich die latente Variable $Q$ in die Nutzenfunktion ein. Der Fehler des Wahlmodells ist durch $\varepsilon$ gegeben. Abhängig von der für den Fehler getroffenen Verteilungsannahme kann der Nutzen über ein Logit- oder Probit-Modell erfasst werden. Die Wahlentscheidung wird ausgedrückt als Funktion des Nutzens, unter Annahme der Nutzenmaximierungstheorie, so dass für den Auswahl-Indikator $y_{i}$ der Alternative $i$ gilt:

$$
y_{i}= \begin{cases}1 & \text { falls } U_{i}=\max \left(U_{j}\right) \\ 0 & \text { sonst }\end{cases}
$$

Der Indikator erhält dann den Wert 1, wenn die betrachtete Alternative $i$ im Vergleich zu allen anderen Alternativen $j$ den größten Nutzen stiftet. Dies würde einem Kausalmodell mit einer (ohne Fehler gemessenen) binär abhängigen Variable entsprechen.

Die gemeinsame Wahrscheinlichkeit der beobachtbaren Variablen $y$ und $I$, bedingt auf die exogenen Variablen $X$ und $Z$ und die latente Variable $Q$, muss über alle Haushalte und über alle Alternativen aufsummiert und dann im Hinblick auf die Parameter maximiert werden. Für die latente Variable ist nur die Verteilung bekannt, so dass die gemeinsame Wahrscheinlichkeit über das latente Konstrukt $Q$ integriert werden muss. Die Zielfunktion eines Hybriden Wahlmodells ist daher durch

\footnotetext{
${ }^{2}$ In der klassischen Strukturgleichungsnotation (LISREL) wird der Störterm als $\zeta$ bezeichnet (vgl. u.a. Hildebrandt \& Temme 2005, Jöreskog \& Sörbom 1996).
} 


$$
\begin{aligned}
& f_{1}\left(y, I \mid X, Z ; \alpha, \beta, \gamma, \Sigma_{v}, \Sigma_{\varepsilon}, \Sigma_{\eta}\right)= \\
& \int_{Q} P\left(y \mid X, Q ; \beta, \Sigma_{\varepsilon}\right) f_{2}\left(I \mid Q ; \alpha, \Sigma_{v}\right) f_{3}\left(Q \mid Z ; \gamma, \Sigma_{\eta}\right)
\end{aligned}
$$

gegeben, wobei $\Sigma_{\varepsilon}, \Sigma_{v}$ und $\Sigma_{\eta}$ die Kovarianz-Matrizen der jeweiligen Fehlerterme sind. Die Auswahlwahrscheinlichkeit $P(\ldots)$ und die Verteilung $f_{2}(\ldots)$ der Indikatoren werden, bedingt auf die latente Variable $Q$, als unabhängig voneinander angenommen, so dass die gemeinsame Verteilung durch das Produkt der marginalen Verteilungen gegeben ist (Ashok et al. 2002). Wie aus Gleichung (5) zu erkennen ist, ergibt sich die gemeinsame Wahrscheinlichkeit $f_{l}(\ldots)$ aus den zwei Bestandteilen des in $A b b .5$ dargestellten Hybriden Wahlmodells.

Es besteht die Möglichkeit, durch Einführung latenter Klassen der Konsumentenheterogenität verstärkt Rechnung zu tragen (Chintagunta et al. 1991; Kamakura/Russell 1989). So werden beispielsweise mit einem Finite Mixture Ansatz für eine begrenzte Anzahl in sich weitgehend homogener Konsumentensegmente, die jedoch für den Marktforscher unbeobachtbar sind, jeweils segmentspezifische Koeffizienten geschätzt (Dillon et al. 1994). Heterogenität kann einen wesentlichen Einfluss auf die Schätzergebnisse in einem Hybriden Wahlmodell besitzen. Um einer möglichen Heterogenität in den Präferenzen der Grundgesamtheit Rechnung zu tragen, kann die in Gleichung (5) angegebenen Zielfunktion angepasst werden. Für den Fall einer diskret verteilten Heterogenität kann ein Modell, das latente Klassen berücksichtigt, beispielsweise das Finite Mixture Logit Modell (Chintagunta et al. 1991; Kamakura/Russell 1989) in das Hybride Wahlmodell integriert werden, so dass die Wahlwahrscheinlichkeit

$$
P\left(y \mid X, Q ; \beta, \Sigma_{\varepsilon}^{s}\right)=\sum_{s=1}^{S}\left(P\left(y \mid X, Q ; \beta^{s}, \Sigma_{\varepsilon}^{s}\right) \Lambda(s \mid X, Q)\right)
$$

ist (vgl. Walker 2001). Dabei stellt $S$ die Anzahl an latenten Segmenten dar und $\Lambda$ die Wahrscheinlichkeit, dem Segment $s$ anzugehören.

Neben klassischen Maximum Likelihood (ML) Verfahren ist die Schätzung durch die Maximum Simulated Likelihood (MSL) Methode, die Method of Simulated Moments (MSM) oder durch bayesianische Verfahren denkbar. Bislang finden sich in der Literatur vor allem Ansätze, die einen MSL-Schätzer nutzen (vgl. Ben-Akiva et al. 2002b). Dabei lassen sich verschiedene Vorteile, die für diese Anwendung sprechen, ausmachen: Zum Einen erleichtert die 
Verwendung eines MSL-Schätzers den Programmieraufwand, da eine verallgemeinerbare Schätzroutine genutzt werden kann. Zum Anderen vereinfacht die MSL-Methode die Integration von mehrdimensionalen Integralen. Dies ist vorteilhaft, da mit der Anzahl an latenten Variablen die Dimension der Zielfunktion steigt. Befinden sich höchstens drei Variablen bzw. Konstrukte im Modell, über die Verteilungsannahmen getroffen werden können, kann die Schätzung mit numerischer Integration mit einem Maximum Likelihood Verfahren durchgeführt werden. Sofern über mehr als drei Dimensionen integriert werden muss, bietet sich der Einsatz von Simulationstechniken an. Die MSL-Schätzung ermöglicht eine Approximation der Zielfunktion über die simulierten Wahrscheinlichkeiten (vgl. u.a. Train 2003). Um vollständig effiziente Schätzer zu erlangen, sollten alle Informationen der Gleichungen (1) - (4) simultan in die Zielfunktion einfließen (Morikawa et al. 2002). Die Schätzung dieser Modelle ist mittels Full-Information (FI)-Verfahren möglich. Diese sind dadurch gekennzeichnet, dass bei der Schätzung alle Parameter, die der postulierten strukturellen Form unterliegen, simultan berücksichtigt werden (Frohn 1995).

Alternativ zu MSL-Schätzern ist es denkbar, einen Simulated Mean of Bayesian Posterior (SMP)-Schätzer zu nutzen (vgl. u.a. Train 2001). Der SMP-Schätzer ist konsistent und effizient unter weniger stringenten Bedingungen als der MSL-Schätzer und verhindert numerische Probleme bei der Bestimmung eines Maximums (vgl. Ben-Akiva et al. 2002b). Der wesentliche Vorteil der Anwendung von bayesianischen Verfahren im Kontext von Hybriden Wahlmodellen besteht darin, dass die multidimensionale Wahlwahrscheinlichkeit nicht durch aufwändige Berechnungen bestimmt werden muss (vgl. Bolduc et al. 2005). Jedoch ist der SMP-Schätzer abhängig von iterativen Stichprobenverfahren, vor allem der Markov Chain Monte Carlo (MCMC)-Methode. Es ist unter Umständen schwer zu bestimmen, ob eine Konvergenz erzielt wurde (vgl. Ben-Akiva et al. 2002a). Ein vertiefender Überblick und ein Vergleich von MSL- und SMP-Verfahren für Hybride Wahlmodelle findet sich in Bolduc et al. (2005).

Verschiedenen Studien (u.a. Train 2001; Bolduc et al. 1997) haben für traditionelle Wahlmodelle gezeigt, dass die Wahl des Schätzverfahrens durch zwei wesentliche Punkte, nämlich den Implementierungsaufwand und die benötigte Rechenzeit, bestimmt werden kann. Train (2003) weist nach, dass für hinreichend viele Beobachtungen beide Methoden (MSL wie auch SMP) unter normalen Bedingungen die gleichen Ergebnisse erzeugen. Dem Anwender ist es damit selbst überlassen, welches Verfahren er zur Schätzung eines Hybriden Wahlmodells wählt. 
In keinem handelsüblichen Softwarepaket ist das Multinomiale Hybride Wahlmodell bislang implementiert. Softwareprogramme wie WinBUGS, GAUSS und MATLAB haben das Potenzial zur Schätzung solcher Modelle, es muss jedoch eine spezifische und individuelle Programmierung durch den Anwender vorgenommen werden. Walker/Ben-Akiva (2002) erläutern in detaillierten Einzelschritten die Entwicklung einer adäquaten Likelihoodfunktion und zeigen in mehreren Fallstudien die Umsetzung der Methodik. Temme (2007) weist mit einer Simulationsstudie nach, dass das Softwarepaket Mplus (Muthén/Muthén 1998-2007) eine elegante Programmierung des HCM erlaubt.

\section{Anwendungsbereiche}

Dem Marketingforscher wird mit Hybriden Wahlmodellen ein leistungsstarkes Instrumentarium zur Verfügung gestellt, mit dem Kausalmodelle und Wahlmodelle in einem gemeinsamen Ansatz vereint werden. Außerdem bieten Hybride Wahlmodelle die Möglichkeit, unterschiedliche Informationsquellen miteinander zu verknüpfen, um realitätsgetreuere Ergebnisse zu erhalten. Hieraus ergeben sich aber auch spezielle Anforderungen an die zu analysierenden Daten (Ben-Akiva et al. 1999). Neben Daten über das tatsächliche Auswahlverhalten (z.B. Markenwahl und Preis aus Scanner-Panel-Daten) sind auch Befragungsdaten zu den Einstellungen und Werten der Entscheidungsträger notwendig, um den Einfluss geeigneter latenter Variablen modellieren zu können. Datenbasen die diesen Anforderungen gerecht werden sind bspw. Konsumentenpanels. Es sei darauf hingewiesen, dass die Implementierung des Hybriden Wahlmodells rechen- und computertechnisch sehr arbeitsintensiv ist. Im Folgenden werden einige empirische Anwendungen des HCM-Ansatzes skizziert.

Walker et al. (2007) befassen sich in diesem Zusammenhang mit einer für das Tourismusmarketing, die Automobilindustrie oder den Öffentlichen Personennahverkehr (so z.B. in der Preis- und Tarifgestaltung sowie der Kommunikationspolitik) relevanten Fragestellung. Es wurde untersucht, inwieweit die latenten Variablen „attitudes“, „social norms“ und „perceived behavioral control“ einen Einfluss auf die Verkehrsmittelwahl haben. Aus einem Verbraucherpanel eines internationalen Marktforschungsdienstleisters wurden 907 Teilnehmer rekrutiert. Zusätzlich zu den klassischen Variablen „accessibility“, „travel time“ etc. wurden auch individuelle Auswahlkriterien und Motivationen der Panelteilnehmer erhoben. Die Auswahlkriterien wurden in einer qualitativen Studie in Fokusgruppen entwickelt und umfassen Konstrukte wie „privacy“, „time“ und „flexibility“. Die Teilnehmer beurteilten die Wichtigkeit 
dieser Auswahlkriterien an 5-stufigen Ratingskalen. Zur Messung der Motivationen wurde der PQ-Fragebogen von Schwartz et al. (1999) herangezogen. Die Teilnehmer bewerteten ihre Ähnlichkeit zu 40 Personenbeschreibungen auf 6-stufigen Ratingskalen. Das spezifizierte Hybride Wahlmodell umfasste sowohl individuelle Auswahlkriterien (flexibility, possession, passivity, environmental protection), als auch Werte bzw. Wertvorstellungen (power, hedonism, security) der Panelteilnehmer. Es konnte u.a. gezeigt werden, dass die Auswahlkriterien von der zugrunde liegenden Wertorientierung bestimmt werden. Ein Chi-QuadratDifferenzentest bestätigte den substanziellen Erklärungskraftzuwachs des Hybriden Wahlmodells und unterstreicht somit die Relevanz der Aufnahme latenter Variablen in ein klassisches Wahlmodell.

Das im Rahmen der explanativen Warenkorbanalyse dominierende klassische Logit- oder Probit-Wahlmodell (vgl. hierzu Boztuğ/Silberhorn 2006) wird von Silberhorn et al. (2007a) um den Einfluss von latenten Konsumentenzielen erweitert. Anhand von Daten aus dem Verbraucherpanel der Gesellschaft für Konsumforschung (GfK) wurde untersucht, inwieweit das Verbundkaufverhalten der Konsumenten durch deren Schlankheitsorientierung beeinflusst wird. Die zielabgeleitete Produktkategorie slim food umfasst die nominalen Produktkategorien Müsli und Quark, die über diese zielabgeleitete Kategorie in Beziehung zueinander stehen. Demnach sind zielabgeleitete Kategorien ein weiterer Faktor, der im Rahmen der Warenkorbanalyse einen Einfluss auf das Verbundkaufverhalten ausüben kann. Eine große Schlankheitsorientierung führt erwartungsgemäß zu einer größeren Wahrscheinlichkeit eines Verbundkaufs in beiden Produktkategorien, d.h. die Vermutung eines positiven signifikanten Einflusses der latenten Variable auf die Kaufentscheidung in den Produktkategorien Müsli und Quark konnte bestätigt werden. Die Verbesserung der Modellgüte spiegelte sich in den Informationskriterien AIC und BIC wieder. Silberhorn et al. (2007b) erweitern diesen Ansatz um eine zweite Hierarchieebene, indem sie ein übergeordnetes Gesundheitsziel mit der Vermeidung von gesundheitlichen Risikofaktoren wie Zucker und Fett in Beziehung setzen und den davon ausgehenden Einfluss auf Produktkaufentscheidungen modellieren. Auch in diesem Fall konnte das Hybride Wahlmodell einen besseren Fit erzielen und ein signifikanter Einfluss des Gesundheitsziels auf die Kaufentscheidungen nachgewiesen werden.

Neben den hier kurz vorgestellten empirischen Anwendungen, kann das Hybride Wahlmodell auch in zahlreichen anderen Gebieten im Marketing zum Einsatz kommen (siehe u.a. Dellaert/Stremersch 2005). Einige wichtige Anwendungsbereiche und mögliche Anwendungsbeispiele im Marketing sind in Tab. 1 aufgeführt. 


\begin{tabular}{|c|c|c|}
\hline $\begin{array}{l}\text { Teilbereich des Mar- } \\
\text { keting }\end{array}$ & Anwendungsbeispiel & Latente Variable \\
\hline Kundenbindung & $\begin{array}{l}\text { - Messung der Kundenzu- } \\
\text { friedenheit }\end{array}$ & $\begin{array}{l}\text { - wahrgenommene Ser- } \\
\text { vicequalität }\end{array}$ \\
\hline Werbung & $\begin{array}{l}\text { - } \text { Rolle der Markenwahr- } \\
\text { nehmung in der Marken- } \\
\text { wahl }\end{array}$ & $\begin{array}{ll}\text { - } & \text { Markenimage } \\
\text { - } & \text { Markenstärke }\end{array}$ \\
\hline Konsumentenverhalten & $\begin{array}{ll}\text { - } & \text { Einfluss von Werten und } \\
\text { Einstellungen auf Kauf- } \\
\text { entscheidungen }\end{array}$ & - Gesundheitsbewusstsein \\
\hline Preispolitik & 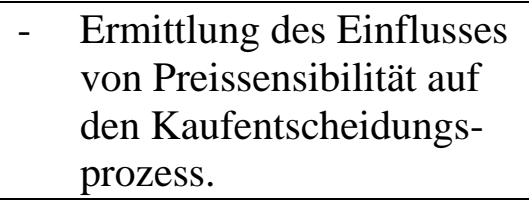 & - Preisbewusstsein \\
\hline Produktpolitik & 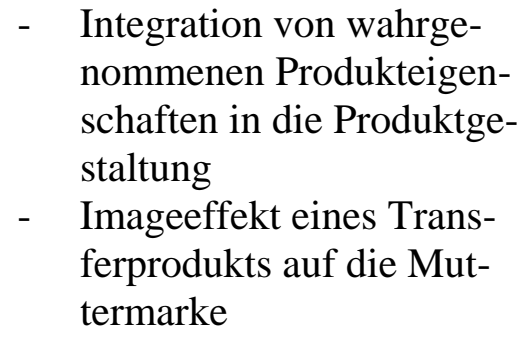 & $\begin{array}{l}\text { - } \quad \begin{array}{l}\text { wahrgenommene Pro- } \\
\text { duktqualität }\end{array} \\
\text { - } \quad \text { Markenpersönlichkeit }\end{array}$ \\
\hline
\end{tabular}

Tab. 1: Anwendungsbereiche des Hybriden Wahlmodells im Marketing

\section{Erweiterungsmöglichkeiten}

Jedes Wahlmodell unter Annahme der Nutzenmaximierungstheorie kann neben der hier diskutierten Aufnahme latenter Variablen auch unter anderen Gesichtspunkten erweitert werden (Walker/Ben-Akiva 2002).

So ist es zunächst möglich, „revealed preferences“ (RP) und „stated preferences“ (SP) in einem Modell zu kombinieren. Revealed preferences repräsentieren tatsächliches Verhalten, stated preferences resultieren aus Befragungen. Eine fundamentale Annahme bei der Durchführung von SP-Befragungen besteht darin, dass die Trade-Off Beziehung zwischen den wichtigen Attributen den revealed und stated preferences gemein ist (Morikawa et al. 2002). Ansonsten käme SP-Befragungen nur eine geringe Bedeutung zu. Für andere Faktoren mit Einfluss auf revealed und stated preferences werden in den RP- und SP-Modellen unterschiedliche Koeffizienten angenommen.

Darüber hinaus können die Verteilungsannahmen der stochastischen Fehlerterme flexibilisiert werden (Bolduc et al. 2005). Eine Möglichkeit besteht darin, den Fehlerterm in zwei additiv ver- 
knüpfte Komponenten aufzuspalten (Mixed Logit Modell): Eine Fehlerkomponente ist über die Auswahlalternativen hinweg korreliert und heteroskedastisch, der andere Teil ist wie im klassischen Logit Modell über Alternativen und Individuen unabhängig und identisch extremwertverteilt (vgl. Hensher/Greene 2003). Auch das Probit Modell, das von normalverteilten und korrelierten Fehlertermen ausgeht, ist modelltechnisch attraktiv, da es im Gegensatz zum Logit Modell frei von der Independence of Irrelevant Alternatives (IIA) Annahme ist. Chintagunta (1992) stellt mit der Method of Simulated Moments (MSM) eine Methode vor, die eine bequeme Schätzung von Probit Modellen erlaubt.

Wie bereits in Gleichung (6) gezeigt, besteht grundsätzlich die Möglichkeit, durch Einführung latenter Klassen der Konsumentenheterogenität verstärkt Rechnung zu tragen (Chintagunta et al. 1991; Kamakura/Russell 1989).

In $A b b .6$ sind die genannten Erweiterungen des konventionellen Wahlmodells grafisch dargestellt. Im Zentrum steht dabei die vertikale Achse des klassischen Wahlmodells.

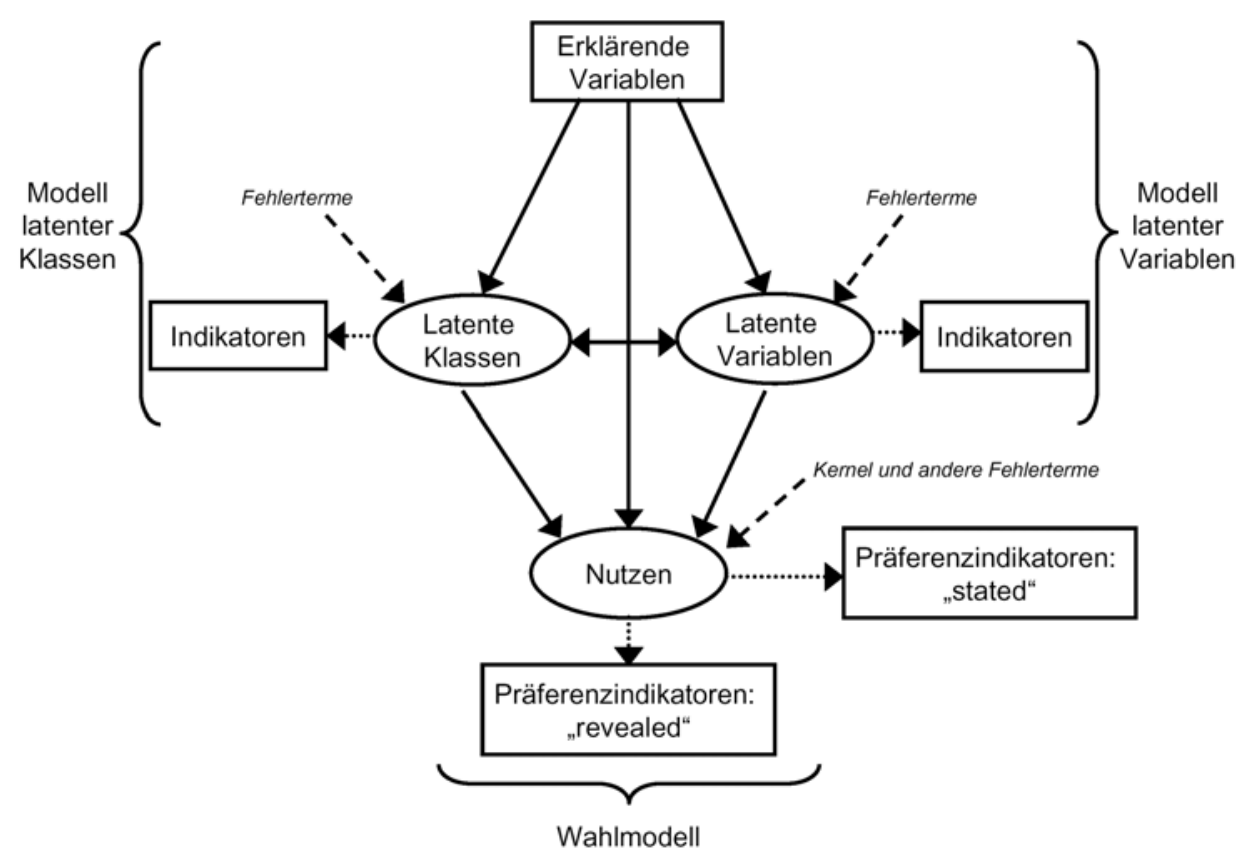

Abb. 6: Generalisiertes Zufallsnutzenmodell (Walker/Ben-Akiva 2002)

Darüber hinaus besteht die Möglichkeit, das Hybride Wahlmodell durch die Berücksichtigung von Endogenität zu erweitern. Im Kontext der aktuellen Wahlmodellforschung wird zunehmend die Berücksichtigung einer möglichen Korrelation zwischen Nachfrage- und Angebotsverhalten gefordert (Louviere et al. 2005). Da insbesondere das Marketing-Mix-Instrument 
Preis zumeist nicht unabhängig vom Nachfrageverhalten festgelegt wird, ergibt sich zwangsläufig eine Beziehung zwischen der als exogen angenommen Größe und dem modellierten Fehlerterm. Die Vernachlässigung einer solchen Beziehung kann unter Umständen zu verzerrten Schätzergebnissen führen (Villas-Boas/Winer 1999). Um dieses Problem zu umgehen, muss das mit dem Fehlerterm korrelierte Marketing-Mix-Instrument als endogene Variable betrachtet werden. Prinzipiell ist die Umsetzung dieses Punktes in einem Hybriden Wahlmodell denkbar. Dazu ist eine zusätzliche Verteilungsannahme über die Beziehung zwischen dem Wahlverhalten und der endogenen Größe in den Modellrahmen zu integrieren. Gegeben die hohe Komplexität der Hybriden Wahlmodelle, ist eine Umsetzung dieses Punktes jedoch bislang noch nicht erfolgt.

Der Einsatz formativer Indikatoren, bei der Spezifikation der latenten Größen wäre ebenfalls vorstellbar, müsste jedoch bezüglich der Umsetzbarkeit insbesondere auf Grund möglicher Identifikationsprobleme (so z.B. Temme 2006) geprüft werden. Es ist jedoch davon auszugehen, dass bei der psychologischen Erklärung des Kaufverhaltens die reflektive Spezifizierung der Messmodelle psychologischer Größen im Vordergrund steht (siehe Jarvis et al. 2003). Für eine Diskussion der Anwendung formativer und reflektiver Indikatoren sei an dieser Stelle außerdem auf Albers/Hildebrandt (2006) sowie Diamantopoulos/Winklhofer (2001) verwiesen.

\section{Zusammenfassung}

Der vorliegende Beitrag liefert eine schrittweise Einführung in das im Marketing bislang weitgehend vernachlässigte Hybride Wahlmodell, eine Methode, die zwei in der Marketingforschung etablierte Verfahren kombiniert.

Strukturgleichungsmodelle haben sich zu einer der meistgenutzten Methoden in den Wirtschafts- und Sozialwissenschaften (z. B. Marketing) entwickelt. Die entscheidende Stärke dieser Modellierung liegt darin, dass die Effekte von Messfehlern zu kontrollieren sind. Neben dieser kausalanalytischen Vorgehensweise spielen gerade im Marketing auch prognoseorientierte Wahlmodelle zur Analyse von Entscheidungsprozessen eine große Rolle. Einfache Wahlmodelle verfolgen primär das Ziel, Richtung und Stärke möglicher Kaufverhaltenstreiber zu schätzen. Der psychologisch begründete Prozess der Kaufentscheidung wird dabei im Wesentlichen als „Black Box“ aufgefasst. Das Hybride Wahlmodell integriert Kausal- und Wahlmodell in einem umfassenden Modellansatz und 
vereint somit die Vorteile beider Verfahren. Durch die Verbindung dieser beiden Methoden kann das Entscheidungsverhalten der Konsumenten verhaltenswissenschaftlich besser erklärt werden. Die vielfältigen Anwendungsmöglichkeiten machen das Hybride Wahlmodell zu einem leistungsstarken Instrument für detailliertere Studien des Konsumentenverhaltens.

Gleichzeitig werden jedoch verschiedene Problembereiche aufgezeigt. Zum Einen gibt es aus methodischer Sicht noch Weiterentwicklungsbedarf; Hinweise hierzu liefert Kapitel 5. Zum Anderen steht die standardmäßige Implementierung des multinomialen Hybriden Wahlmodells in handelsüblicher Software noch aus. Einzig das Softwarepaket Mplus (Muthén/Muthén 1998-2007) erlaubt bislang eine elegante Programmierung. Natürlich ist es auch möglich, die zur Ermittlung der Koeffizientenschätzer notwendige Zielfunktion in einer geeigneten Optimierungssoftware selbst zu implementieren. 


\section{Literaturverzeichnis}

Ainslie, A./Rossi, P.E. (1998): Similarities in Choice Behavior Across Product Categories, in: Marketing Science, Vol. 17, No. 2, pp. 91-106.

Albers, S./Hildebrandt, L. (2006): Methodische Probleme bei der Erfolgsfaktorenforschung Messfehler, formative versus reflektive Indikatoren und die Wahl des Strukturgleichungs-Modells, in: zfbf, Februar 2006, S. 2-33.

Ashok, K./Dillon, W. R./Yuan, S. (2002): Extending Discrete Choice Models to Incorporate Attitudinal and Other Latent Variables, in: Journal of Marketing Research, Vol. 39, No. 1, pp. 31-46.

Ben-Akiva, M./Lerman, S.R. (1985): Discrete Choice Analysis: Theory and Application to Travel Demand, $6^{\text {th }}$ ed., Cambridge/Massachussetts.

Ben-Akiva, M./McFadden, D. L./Gärling, T./Gopinath, D. A./Walker, J. L./Bolduc, D./BörschSupan, A./Delquié, P./Larichev, O./Morikawa, T./Polydoropoulou, A./Rao, V. (1999): Extended Framework for Modeling Choice Behavior, in: Marketing Letters, Vol. 10, No. 3, pp. 187-203.

Ben-Akiva, M./McFadden, D. L./Train, K./Walker, J. L./Bhat, C./Bierlaire, M./Bolduc, D./Börsch-Supan, A./Brownstone, D./Bunch, D. S./Daly, A./de Palma, A./Gopinath, D. A./Karlstrom, A./Munizaga, M. A. (2002a): Hybrid Choice Models: Progress and Challenges, in: Marketing Letters, Vol. 13, No. 3, pp. 163-175.

Ben-Akiva, M./Walker, J. L./Bernardino, A. T./Gopinath, D. A./Morikawa, T./Polydoropoulou, A. (2002b) : Integration of Choice and Latent Variable Models. In: H. S. Mahmassani (Ed.): In Perpetual Motion: Travel Behavior Research Opportunities and Application Challenges, Oxford, pp. 431-470.

Blackwell, R. D./Miniard, P. W:/Engel, J. F. (2006): Consumer Behavior, 10th ed., Mason/Ohio, USA.

Bolduc, D./Fortin, B./ Gordon, S. (1997): Multinomial Probit Estimation of Spatially Interdependent Choices: An Empirical Comparison of Two New Techniques, in: International Regional Science Review, Vol. 20, No. 1\&2, pp. 77-101.

Bolduc, D./Ben-Akiva, M./Walker, J. L./Michaud, A. (2005): Hybrid Choice Models with Logit Kernel: Applicability to Large Scale Models, Working Paper, Université Laval, Québec, Canada. 
Boztuğ, Y./Silberhorn, N. (2006): Modellierungsansätze in der Warenkorbanalyse im Überblick, in: Journal für Betriebswirtschaft, Vol. 56, S. 105-126.

Chib, S./Greenberg, E. (1998): Analysis of Multivariate Probit Models, in: Biometrika, Vol. 85, No. 2, pp. 347-361.

Chintagunta, P. K. (1992): Estimating a multinomial probit model of brand choice using the method of simulated moments, in: Marketing Science, Vol. 11, No. 4, pp. 386-407.

Chintagunta, P. K. (1993): Investigating Purchase Incidence, Brand Choice and Purchase Quantity Decisions of Households, in: Marketing Science, Vol. 12, No. 2, pp. 184-208.

Chintagunta P. K./Jain, D. C./Vilcassim, N. J (1991): Investigating heterogeneity in brand preferences in logit models for panel data, in: Journal of Marketing Research, Vol. 28, No. 4, pp. 417-428.

Dellaert, B. G. C./Stremersch, S. (2005): Marketing Mass-Customized Products: Striking a Balance Between Utility and Complexity, in: Journal of Marketing Research, Vol. 42, No. 2, pp. 219-227.

Dillon, W. R./Böckenholt, U./Smith de Borrero, M./Bozdogan, H./de Sarbo, W./Gupta, S./Kamakura, W./Kumar, A./Ramaswamy, B./Zenor, M. (1994): Issues in the Estimation and Application of Latent Structure Models of Choice, in: Marketing Letters, Vol. 5, No. 4, pp. 323-334.

Diamantopoulos, A./Winklhofer, H. M. (2001): Index Construction with Formative Indicators: Alternative to Scale Development, in: Journal of Marketing Research, Vol. 38, No. 2, pp. 269-277.

Elrod, T./Keane, M. P. (1995): A Factor-Analytic Probit Model for Representing the Market Structure in Panel Data, in: Journal of Marketing Research, Vol. 32, No.1, pp. 1-16.

Frohn, J. (1995): Grundausbildung in Ökonometrie, 2. Aufl., Berlin.

Gopinath, D. A. (1995): Modelling Heterogeneity in Discrete Choice Processes: Application to Travel Demand, PhD Thesis, Cambridge, MA.

Gopinath, D. A./Schofield, M. L./Walker, J. L./Ben-Akiva, M. (2004): Comparative Analysis of Discrete Choice Models with Flexible Substitution Patterns, Working Paper, Massachusetts Institute of Technology, Cambridge/Massachusetts, USA.

Guadagni, P. M./Little, J. D. C. (1983): A Logit Model of Brand Choice Calibrated in Scanner Data, in: Marketing Science, Vol. 2, No. 3, pp. 203-238. 
Hausman, J.A./Wise, D.A (1978): A conditional probit model for qualitative choice: Discrete decisions recognizing interdependence and heterogeneous preferences, in: Econometrica, Vol. 46, No. 2, pp. 403-426.

Hensher, D. A./Greene, W. H. (2003): The Mixed Logit model: The state of practice, in: Transportation, Vol. 30, pp. 133-176.

Hildebrandt, L./Homburg, C. (ed.) (1998): Die Kausalanalyse, Stuttgart.

Hildebrandt, L./Temme, D. (2005): Die Modellierung von Problemen der Marketingforschung mit Strukturgleichungsmodellen, in: Haas, A./Ivens, B.S. (eds.): Innovatives Marketing, Gabler Wiesbaden, pp. 49-70.

Hruschka, H./Lukanowicz, M./Buchta, C. (1999): Cross-category sales promotion effects, in: Journal of Retailing and Consumer Services, Vol. 6, pp. 99-105.

Jarvis, C. B./MacKenzie, S. B./Podsakoff, P. M. (2003): A Critical Review of Construct Indicators and Measurement Model Misspecification in Marketing and Consumer Research, in: Journal of Consumer Research, Vol. 30, pp. 199-218.

Jöreskog, K.G./Sörbom, D. (1996): LISREL 8: User’s Reference Guide, Chicago.

Johansson, M.V./Heldt, T./Johansson, P. (2005): Latent Variables in a Travel Mode Choice Model: Attitudinal and Behavioural Indicator Variables. Working Paper 2005:5, Uppsala University.

Kamakura, W. A./Russell, G. J. (1989): A Probabilistic Choice Model for Market Segmentation and Elasticity Structure, in: Journal of Marketing Research, Vol. 26, pp. 379-390.

Keane, M. P. (1997): Modeling Heterogeneity and State Dependence in Consumer Choice Behavior, in: Journal of Business and Economic Statistics, Vol. 15, No. 3, pp. 310-327.

Koppelman, F./Hauser, J. (1979): Destination Choice for Non-Grocery-Shopping Trips, in: Transportation Research Record, Vol. 673, pp. 157-165.

Louviere, J./ Train, K./ Ben-Akiva, M./ Bhat, C./ Brownstone, D./ Cameron, T./ Carson, R./ Deshazo, J./ Fiebig, D./ Greene, W./ Hensher, D. \& Waldman, D. (2005): Recent Progress on Endogeneity in Choice Modeling, in: Marketing Letters, Vol. 16, No. 3-4, pp. 255-265.

Madana, S. M./ Yang, C. Y. D./ Yen, Y.-M. (1995): Analysis of Stated Route Diversion Intentions Under Advanced Traveler Information Systems Using Latent Variable Modeling, in: Transportation Research Record, Vol. 1485, pp. 10-17. 
McFadden, D. L. (1974): Conditional logit analysis of qualitative choice behavior, in: Zarembka, P.: Frontiers in econometrics, New York/London, pp. 105-142.

McFadden, D. L. (1980): Econometric Models for Probabilistic Choice among Products, in: The Journal of Business, Vol. 53, No. 3.2, pp. 13-29.

McFadden, D. L. (1986): The Choice Theory Approach to Marketing Research, in: Marketing Science, Vol. 5, No. 4, pp. 275-297.

McFadden, D. L. (1997): Rationality for Economists. Presented at the NSF Symposium on Eliciting Preferences, Berkeley/California, July.

McFadden, D. L. (2000): Economic Choices, Nobel Prize Lecture, December 8.

Morikawa, T. (1989): Incorporating Stated Preference Data in Travel Demand Analysis, Ph.D.

Dissertation, Department of Civil and Environmental Engineering, Massachusetts Institute of Technology, Cambridge/Massachusetts, USA.

Morikawa, T./Ben-Akiva, M./McFadden, D. L. (2002): Discrete Choice Models Incorporating Revealed Preferences and Psychometric Data, in: Franses, P. H./Montgomery, A. L.: Econometric Models in Marketing, pp. 29-55.

Muthén, L. K./Muthén, B. O. (1998-2007): Mplus User’s Guide, 4th ed., Los Angeles, CA.

Muthén, B. O. (2002): Beyond SEM: General Latent Variable Modeling, in: Behaviormetrika, Vol. 29, No. 1, pp. 81 - 117

Niraj, R./Padmanabhan, V./Seetharaman, P.B. (2007): A Cross-Category Model of Households’ Incidence and Quantity Decisions, in: Marketing Science, conditionally accepted. Russell, G. J./Petersen, A. (2000): Analysis of Cross Category Dependence in Market Basket Selection, in: Journal of Retailing, Vol. 76, No.3, pp. 367-392.

Schwartz, S. H./Lehmann, A./Roccas, S. (1999). Multimethod Probes of Basic Human Values, in: J. Adamopolos/Y. Kashima (Eds.): Social psychology and cultural context: Essays in Honour of Harry C. Triandis, Newbury Park, CA, pp. 107-123.

Seetharaman, P. B./Ainslie, A./Chintagunta, P. K. (1999): Investigating Household State Dependence Effects Across Categories, in: Journal of Marketing Research, Vol. 36, pp. 488-500.

Silberhorn, N./Walker, J. L./Hildebrandt, L. (2007a): The Influence of Consumer Goals on Multi-Category Purchase Incidence Decisions, in: Flexible Marketing in an unpredictable World, Proceedings of the $36^{\text {th }}$ EMAC conference, Reykjavik/Iceland, May 2007. 
Silberhorn, N./Temme, D./Hildebrandt, L./ Walker, J. L. (2007b): How Do Consumer Goals Influence Purchase Decisions?, Working Paper, Humboldt-Universität zu Berlin.

Song, I./Chintagunta, P. K. (2007): A Discrete/Continuous Model for Multi-Category Purchase Behavior of Households, in: Journal of Marketing Research, forthcoming.

Temme, D. (2006): Die Spezifikation und Identifikation formativer Meßmodelle der Marketingforschung in Kovarianzstrukturanalysen, in: Marketing ZFP, 28. Jg., Nr. 3, S. 183209.

Temme, D. (2007): Estimation of Hybrid Choice Models with Mplus - A Monte Carlo Study, Arbeitspapier, Humboldt-Universität zu Berlin.

Train, K. (2001): A Comparison of Hierarchical Bayes and Maximum Simulated Likelihood for Mixed Logit, Working Paper, Berkeley, CA.

Train, K. (2003): Discrete Choice Methods with Simulation, Cambridge, United Kingdom.

Villas-Boas, J. M./Winer, R. S. (1999): Endogeneity in Brand Choice Models, in: Management Science, Vol. 45, No. 10, pp. 1324-1338.

Walker, J. L. (2001): Extended Discrete Choice Models: Integrated Framework, Flexible Error Structures, and Latent Variables, PhD Thesis, Cambridge, MA.

Walker, J. L./Ben-Akiva, M. (2002): Generalized random utility model, in: Mathematical Social Sciences, Vol. 43, No. 3, pp. 303-343.

Walker, J. L./Dannewald, T./Paulssen, M./Temme, D. (2007): Hybrid Choice Models - Estimation Using Canned SEM Software. In: Flexible Marketing in an unpredictable World, Proceedings of the $36^{\text {th }}$ EMAC conference, Reykjavik/Iceland, May 2007. 


\section{SFB 649 Discussion Paper Series 2007}

For a complete list of Discussion Papers published by the SFB 649, please visit http://sfb649.wiwi.hu-berlin.de.

001 "Trade Liberalisation, Process and Product Innovation, and Relative Skill Demand" by Sebastian Braun, January 2007.

002 "Robust Risk Management. Accounting for Nonstationarity and Heavy Tails" by Ying Chen and Vladimir Spokoiny, January 2007.

003 "Explaining Asset Prices with External Habits and Wage Rigidities in a DSGE Model." by Harald Uhlig, January 2007.

004 "Volatility and Causality in Asia Pacific Financial Markets" by Enzo Weber, January 2007.

005 "Quantile Sieve Estimates For Time Series" by Jürgen Franke, JeanPierre Stockis and Joseph Tadjuidje, February 2007.

006 "Real Origins of the Great Depression: Monopolistic Competition, Union Power, and the American Business Cycle in the 1920s" by Monique Ebell and Albrecht Ritschl, February 2007.

007 "Rules, Discretion or Reputation? Monetary Policies and the Efficiency of Financial Markets in Germany, 14th to 16th Centuries" by Oliver Volckart, February 2007.

008 "Sectoral Transformation, Turbulence, and Labour Market Dynamics in Germany" by Ronald Bachmann and Michael C. Burda, February 2007.

009 "Union Wage Compression in a Right-to-Manage Model" by Thorsten Vogel, February 2007.

010 "On $\sigma$-additive robust representation of convex risk measures for unbounded financial positions in the presence of uncertainty about the market model" by Volker Krätschmer, March 2007.

011 "Media Coverage and Macroeconomic Information Processing" by Alexandra Niessen, March 2007.

012 "Are Correlations Constant Over Time? Application of the CC-TRIG -test $^{-}$ to Return Series from Different Asset Classes." by Matthias Fischer, March 2007.

013 "Uncertain Paternity, Mating Market Failure, and the Institution of Marriage" by Dirk Bethmann and Michael Kvasnicka, March 2007.

014 "What Happened to the Transatlantic Capital Market Relations?" by Enzo Weber, March 2007.

015 "Who Leads Financial Markets?" by Enzo Weber, April 2007.

016 "Fiscal Policy Rules in Practice" by Andreas Thams, April 2007.

017 "Empirical Pricing Kernels and Investor Preferences" by Kai Detlefsen, Wolfgang Härdle and Rouslan Moro, April 2007.

018 "Simultaneous Causality in International Trade" by Enzo Weber, April 2007.

019 "Regional and Outward Economic Integration in South-East Asia" by Enzo Weber, April 2007.

020 "Computational Statistics and Data Visualization" by Antony Unwin, Chun-houh Chen and Wolfgang Härdle, April 2007.

021 "Ideology Without Ideologists" by Lydia Mechtenberg, April 2007.

022 "A Generalized ARFIMA Process with Markov-Switching Fractional Differencing Parameter" by Wen-Jen Tsay and Wolfgang Härdle, April 2007.

\section{SFB 649, Spandauer Straße 1, D-10178 Berlin} http:/ / sfb649.wiwi.hu-berlin.de

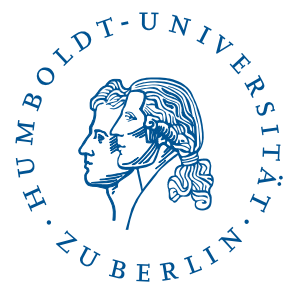


023 "Time Series Modelling with Semiparametric Factor Dynamics" by Szymon Borak, Wolfgang Härdle, Enno Mammen and Byeong U. Park, April 2007.

024 "From Animal Baits to Investors' Preference: Estimating and Demixing of the Weight Function in Semiparametric Models for Biased Samples" by Ya'acov Ritov and Wolfgang Härdle, May 2007.

025 "Statistics of Risk Aversion" by Enzo Giacomini and Wolfgang Härdle, May 2007.

026 "Robust Optimal Control for a Consumption-Investment Problem" by Alexander Schied, May 2007.

027 "Long Memory Persistence in the Factor of Implied Volatility Dynamics" by Wolfgang Härdle and Julius Mungo, May 2007.

028 "Macroeconomic Policy in a Heterogeneous Monetary Union" by Oliver Grimm and Stefan Ried, May 2007.

029 "Comparison of Panel Cointegration Tests" by Deniz Dilan Karaman Örsal, May 2007.

030 "Robust Maximization of Consumption with Logarithmic Utility" by Daniel Hernández-Hernández and Alexander Schied, May 2007.

031 "Using Wiki to Build an E-learning System in Statistics in Arabic Language" by Taleb Ahmad, Wolfgang Härdle and Sigbert Klinke, May 2007.

032 "Visualization of Competitive Market Structure by Means of Choice Data" by Werner Kunz, May 2007.

033 "Does International Outsourcing Depress Union Wages? by Sebastian Braun and Juliane Scheffel, May 2007.

034 "A Note on the Effect of Outsourcing on Union Wages" by Sebastian Braun and Juliane Scheffel, May 2007.

035 "Estimating Probabilities of Default With Support Vector Machines" by Wolfgang Härdle, Rouslan Moro and Dorothea Schäfer, June 2007.

036 "Yxilon - A Client/Server Based Statistical Environment" by Wolfgang Härdle, Sigbert Klinke and Uwe Ziegenhagen, June 2007.

037 "Calibrating CAT Bonds for Mexican Earthquakes" by Wolfgang Härdle and Brenda López Cabrera, June 2007.

038 "Economic Integration and the Foreign Exchange" by Enzo Weber, June 2007.

039 "Tracking Down the Business Cycle: A Dynamic Factor Model For Germany 1820-1913" by Samad Sarferaz and Martin Uebele, June 2007.

040 "Optimal Policy Under Model Uncertainty: A Structural-Bayesian Estimation Approach" by Alexander Kriwoluzky and Christian Stoltenberg, July 2007.

041 "QuantNet - A Database-Driven Online Repository of Scientific Information" by Anton Andriyashin and Wolfgang Härdle, July 2007.

042 "Exchange Rate Uncertainty and Trade Growth - A Comparison of Linear and Nonlinear (Forecasting) Models" by Helmut Herwartz and Henning Weber, July 2007.

043 "How do Rating Agencies Score in Predicting Firm Performance" by Gunter Löffler and Peter N. Posch, August 2007.

\section{SFB 649, Spandauer Straße 1, D-10178 Berlin} http:/ / sfb649.wiwi.hu-berlin.de

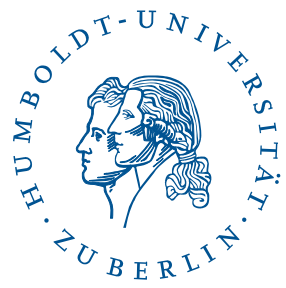


044 "Ein Vergleich des binären Logit-Modells mit künstlichen neuronalen Netzen zur Insolvenzprognose anhand relativer Bilanzkennzahlen" by Ronald Franken, August 2007.

045 "Promotion Tournaments and Individual Performance Pay" by Anja Schöttner and Veikko Thiele, August 2007.

046 "Estimation with the Nested Logit Model: Specifications and Software Particularities" by Nadja Silberhorn, Yasemin Boztuğ and Lutz Hildebrandt, August 2007.

047 "Risiken infolge von Technologie-Outsourcing?" by Michael Stephan, August 2007.

048 "Sensitivities for Bermudan Options by Regression Methods" by Denis Belomestny, Grigori Milstein and John Schoenmakers, August 2007.

049 "Occupational Choice and the Spirit of Capitalism" by Matthias Doepke and Fabrizio Zilibotti, August 2007.

050 "On the Utility of E-Learning in Statistics" by Wolfgang Härdle, Sigbert Klinke and Uwe Ziegenhagen, August 2007.

051 "Mergers \& Acquisitions and Innovation Performance in the Telecommunications Equipment Industry" by Tseveen Gantumur and Andreas Stephan, August 2007.

052 "Capturing Common Components in High-Frequency Financial Time Series: A Multivariate Stochastic Multiplicative Error Model" by Nikolaus Hautsch, September 2007.

053 "World War II, Missing Men, and Out-of-wedlock Childbearing" by Michael Kvasnicka and Dirk Bethmann, September 2007.

054 "The Drivers and Implications of Business Divestiture - An Application and Extension of Prior Findings" by Carolin Decker, September 2007.

055 "Why Managers Hold Shares of Their Firms: An Empirical Analysis" by Ulf von Lilienfeld-Toal and Stefan Ruenzi, September 2007.

056 "Auswirkungen der IFRS-Umstellung auf die Risikoprämie von Unternehmensanleihen - Eine empirische Studie für Deutschland, Österreich und die Schweiz" by Kerstin Kiefer and Philipp Schorn, September 2007.

057 "Conditional Complexity of Compression for Authorship Attribution" by Mikhail B. Malyutov, Chammi I. Wickramasinghe and Sufeng Li, September 2007.

058 "Total Work, Gender and Social Norms" by Michael Burda, Daniel S. Hamermesh and Philippe Weil, September 2007.

059 "Long-Term Orientation in Family and Non-Family Firms: a Bayesian Analysis" by Jörn Hendrich Block and Andreas Thams, October 2007

060 "Kombinierte Liquiditäts- und Solvenzkennzahlen und ein darauf basierendes Insolvenzprognosemodell für deutsche GmbHs" by Volodymyr Perederiy, October 2007

061 "Embedding $\mathrm{R}$ in the Mediawiki" by Sigbert Klinke and Olga ZlatkinTroitschanskaia, October 2007

062 "Das Hybride Wahlmodell und seine Anwendung im Marketing" by Till Dannewald, Henning Kreis and Nadja Silberhorn, November 2007

\section{SFB 649, Spandauer Straße 1, D-10178 Berlin http:/ / sfb649.wiwi.hu-berlin.de}

This research was supported by the Deutsche

Forschungsgemeinschaft through the SFB 649 "Economic Risk".

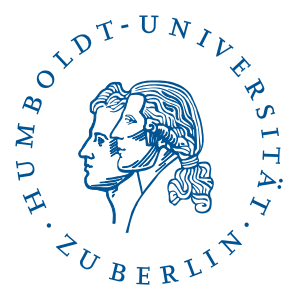

